after annealing to $20 \mathrm{~K}, \ln R \sim T^{-1 / 4}$ was found. These films did not exhibit superconductivity as measured resistively down to the lowest temperatures attainable $(T \sim 1.5 \mathrm{~K})$.

The above-described behavior suggests that the $\mathrm{Hg}-\mathrm{Xe}$ system exhibits a metal-nonmetal transition which occurs with a dependence on concentration and with a critical concentration close to that of continuous percolation in 3D. Beyond the percolation threshold, the systems acquire a negative TCR but are still superconductors. With further increase in Xe concentration, a regime in which the conductivity is dominated by hopping is entered. The approach to an insulating configuration beyond the percolation threshold is probably the Mott-Anderson transition of Refs. 2-4 and is accompanied by the eventual disappearance of superconductivity as determined resistively.

The authors would like to thank R. Mikkelson for many helpful discussions. This work was supported by the U. S. Department of Energy under Contract No. EY-76-S-02-1569-A002.

${ }^{1}$ D. J. Phelps and C. P. Flynn, Phys. Rev. B 14,5279 (1976), and references therein.
${ }^{2}$ Baruch Baz, Aharon Gedanken, Uzi Even, and Joshua Jortner, Phys. Rev. Lett. 28, 1643 (1972).

${ }^{3}$ O. Chesnovski, U. Even, and J. Jortner, Solid State Commun. 22, 745 (1977).

${ }^{4}$ Ori Chesnovski, Uzi Even, and Joshua Jortner, Phys. Lett. 71A, 255 (1979)

${ }^{5}$ B. Abeles and J. J. Hanak, Phys. Lett. $\underline{34 \mathrm{~A},} 165$

(1971).

${ }^{6}$ V. L. Tsymbalenko and A. I. Shal'nikov, Zh. Eksp. Teor. Fiz. $\underline{65}, 2068$ (1973) [Sov. Phys. JETP $\underline{38}, 1043$ (1974)].

${ }^{7}$ N. A. McNeal and A. M. Goldman, J. Low Temp. Phys. 31, 863 (1978).

${ }^{8}$ L. L. Levenson, Nuovo Cimento Suppl. $\underline{5}, 321$ (1967).

${ }^{9}$ K. S. Shante and S. Kirkpatrick, Adv. Phys. 20, 325 (1971).

${ }^{10}$ Hagen Bülow and Werner Buckel, Z. Phys. 145, 141 (1956).

${ }^{11} \mathrm{~J}$. E. Schirber and C. A. Swenson, Phys. Rev. Lett. 2, 296 (1959), and Phys. Rev. 123, 1115 (1961).

${ }^{12}$ K. Epstein, E. D. Dahlberg, R. Mikkelson, and A. M. Goldman, to be published.

${ }^{13}$ C. S. Whitehead, Proc. Roy. Soc. London, Ser. A 238, 175 (1956).

${ }^{14}$ I. O. Kulik and E. Abrahams, to be published.

${ }^{15}$ I. O. Kulik, Pis'ma Zh. Eksp. Teor. Fiz. 14, 341 (1971) [JETP Lett. 14, 228 (1971)].

${ }^{16}$ B. I. Halperin, T. C. Lubensky, and Shang-keng Ma, Phys. Rev. Lett. 32, 292 (1974).

${ }^{17}$ D. G. Naugle, J. W. Baker, and R. E. Allen, Phys. Rev. B $\underline{7}, 3028$ (1973).

\title{
Superconductivity in the Presence of Strong Pauli Paramagnetism: $\mathrm{CeCu}_{2} \mathrm{Si}_{2}$
}

\author{
F. Steglich \\ Institut für Festkörperphysik, Technische Hochschule Darmstadt, D-6100 Darmstadt, West Germany \\ and \\ J. Aarts, C. D. Bredl, W. Lieke, D. Meschede, and W. Franz \\ II. Physikalisches Institut, Universität zu Köln, D-5000 Köln 41, West Germany \\ and \\ H. Schäfer \\ Eduard-Zintl-Institut, Technische Hochschule Darmstadt, D-6100 Darmstadt, West Germany \\ (Received 10 August 1979; revised manuscript received 7 November 1979) \\ A comparison was made between four low-temperature properties of $\mathrm{LaCu}_{2} \mathrm{Si}_{2}$ and \\ $\mathrm{CeCu}_{2} \mathrm{Si}_{2}$. Whereas $\mathrm{LaCu}_{2} \mathrm{Si}_{2}$ behaves like a normal metal, $\mathrm{CeCu}_{2} \mathrm{Si}_{2}$ shows (i) low-tem- \\ perature anomalies typical of "unstable $4 f$ shell" behavior and (ii) a transition into a \\ superconducting state at $T_{c} \approx 0.5 \mathrm{~K}$. Our experiments demonstrate for the first time \\ that superconductivity can exist in a metal in which many-body interactions, probably \\ magnetic in origin, have strongly renormalized the properties of the conduction-elec- \\ tron gas.
}

The relationship between different collective phenomena in metals has continued to interest both experimentalists and theorists. Recent interest has shifted to materials in which ferromagnetism and superconductivity occur at different temperatures, either because of the addition 
of magnetic impurities to a superconducting host ${ }^{1}$ or because the magnetism is intrinsic to the localized $4 f$ electrons of a rare-earth constituent, such as $\mathrm{Er}$, in a superconducting compound. ${ }^{2}$ There is also much interest in materials in which either superconductivity or magnetism interferes with a third kind of collective phenomenon, i.e., the Kondo or intermediate-valence phenomenon, which occurs in metals containing rare earths with less localized $4 f$ electrons, such as Ce. It results from an "instability of the $4 f$ shell" (namely, of the $4 f$ magnetic moment and sometimes also of the $4 f$ occupation number) and is characterized by distinct low-temperature anomalies in the magnetic and electronic transport properties. While Ce impurities can strongly influence the intrinsic properties of a superconducting host, ${ }^{3}$ in certain Ce compounds, e.g., $\mathrm{CeAl}_{2}$, a Kondotype phenomenon seems to coexist with longrange antiferromagnetism. ${ }^{4}$

In this Letter, we report low-temperature observations of the resistivity, specific heat, lowfield ac susceptibility, and dc magnetization of $\mathrm{CeCu}_{2} \mathrm{Si}_{2}$ and $\mathrm{LaCu}_{2} \mathrm{Si}_{2}$. Whereas $\mathrm{LaCu}_{2} \mathrm{Si}_{2}$ shows rather normal metallic behavior, we conclude that in $\mathrm{CeCu}_{2} \mathrm{Si}_{2}$, a compound with "unstable $4 f$ shell" behavior, the low-temperature anomalies reported before by Franz et al. ${ }^{5}$ have their origin, in our somewhat more carefully prepared samples, in a transition into a novel superconducting state. We conclude that a large fraction (up to $30 \mathrm{vol} \%$ ) of the bulk of our $\mathrm{CeCu}_{2} \mathrm{Si}_{2}$ samples is

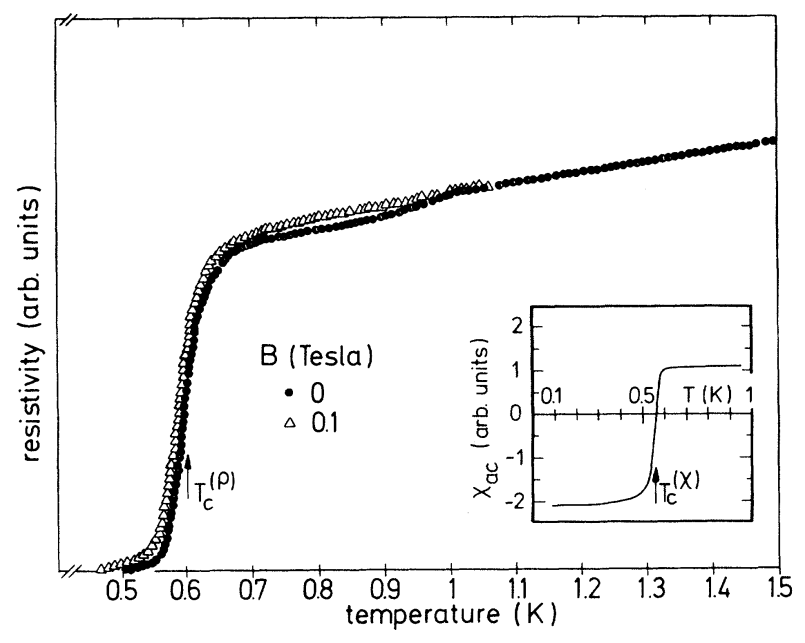

FIG. 1. Resistivity (main part) and low-field ac susceptibility (inset) of $\mathrm{CeCu}_{2} \mathrm{Si}_{2}$ as function of temperature. Arrows give transition temperatures $T_{c}{ }^{(\rho)}=0.60 \pm 0.03$ $\mathrm{K}$ and $\boldsymbol{T}_{c}{ }^{(X)}=0.54 \pm 0.03 \mathrm{~K}$. Transition widths are taken between $10 \%$ and $90 \%$ points of the transition curves. exhibiting the Meissner effect. A preliminary report on some of our results has been given elsewhere. ${ }^{6}$

The polycrystalline samples were prepared in an induction furnance, while kept under an argon pressure of $5 \mathrm{~atm}$. While most results reported here were obtained with unannealed samples, one sample was reinvestigated after annealing in an ultrahigh vacuum $\left(900^{\circ} \mathrm{C}, 100 \mathrm{~h}\right)$. X-ray analysis indicated that both compounds had the proper structure (tetragonal, $\mathrm{ThCr}_{2} \mathrm{Si}_{2}$ ); microprobe analysis, however, revealed the existence of a small amount of precipitations (varying from sample to sample between 1 and 4 vol \%) of both a $\mathrm{Si}-\mathrm{rich}$ phase and a $\mathrm{Cu}-\mathrm{Si}$ phase with a $\mathrm{Cu}$ content of 80-90 at.\%. Upon annealing, no significant change either of the $x$-ray pattern or the microprobe result was detected.

The experimental results of the resistivity, ac susceptibility, and specific heat for an unannealed $\mathrm{CeCu}_{2} \mathrm{Si}_{2}$ sample are presented in Figs. 1 and 2

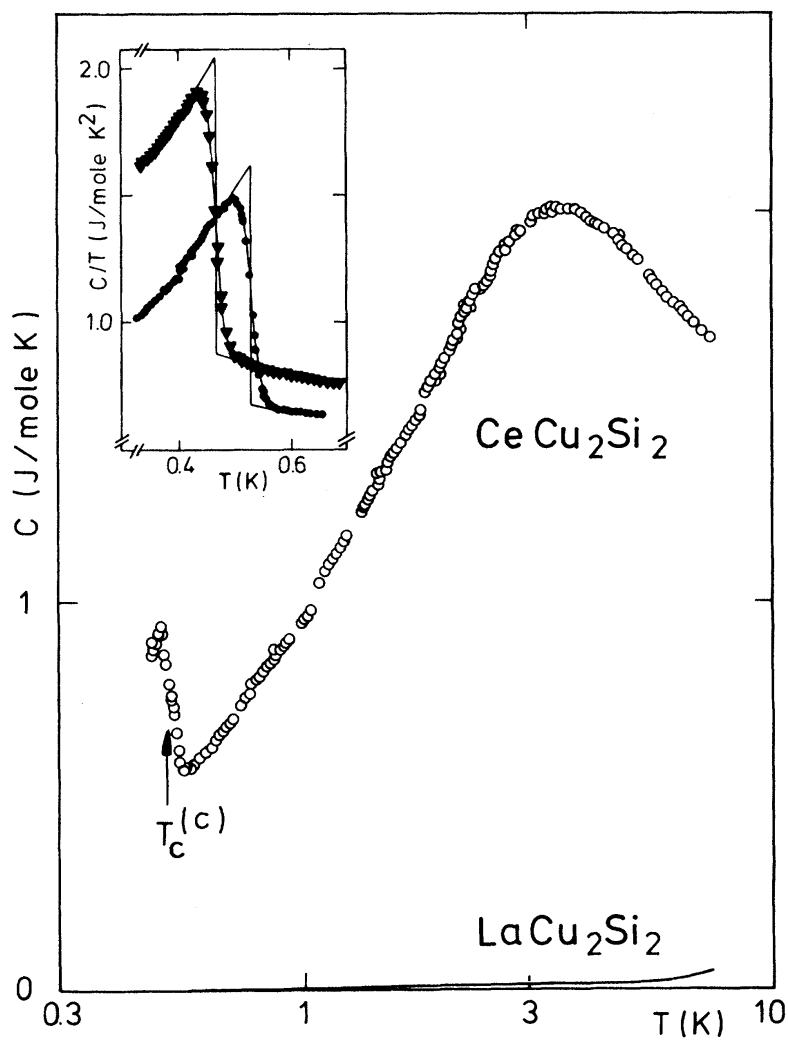

FIG. 2. Molar specific heat of $\mathrm{CeCu}_{2} \mathrm{Si}_{2}$ at $B=0$ as function of temperature on logarithmic scale. Arrow marks transition temperature $T_{c}{ }^{(C)}=0.51 \pm 0.04 \mathrm{~K}$. Transition width determined as in Fig. 1. Inset shows in a $C / T$ vs $T$ plot the specific-heat jumps of two other $\mathrm{CeCu}_{2} \mathrm{Si}_{2}$ samples. 
(main part). We discuss the results for $T>0.6$ $\mathrm{K}$ first. Whereas $\mathrm{LaCu}_{2} \mathrm{Si}_{2}$ behaves like a normal $d$-band metal, $\mathrm{CeCu}_{2} \mathrm{Si}_{2}$ shows several anomalies: (i) Below $1 \mathrm{~K}$, the ac susceptibility is positive and almost temperature independent (inset of Fig. 1); (ii) below $1.5 \mathrm{~K}$, the resistivity increases linearly with $T$ (main part of Fig. 1); (iii) the specific heat has a broad peak $\left(T_{\max }=3.5 \mathrm{~K}\right)$, whose low-temperature $(T<1 \mathrm{~K})$ part is predominated by a $\gamma T$ law with a large coefficient $\gamma \simeq 1$ $\mathrm{J}\left(\mathrm{K}^{2} \text { mole }\right)^{-1}$ (main part of Fig. 2). The resistivity, which was consistent from sample to sample, is displayed in arbitrary units: Determination of the sample geometry was unreliable because of the presence of microcracks in the $\mathrm{CeCu}_{2} \mathrm{Si}_{2}$ samples. ${ }^{5}$

The properties of $\mathrm{CeCu}_{2} \mathrm{Si}_{2}$ between 0.6 and $1 \mathrm{~K}$ are similar to those reported by Andres, Graebner, and $\mathrm{Ott}^{7}$ for $\mathrm{CeAl}_{3}$ in the same temperature regime. ${ }^{8}$ In addition, like $\mathrm{CeAl}_{3},{ }^{9} \mathrm{CeCu}_{2} \mathrm{Si}_{2}$ exhibits a pronounced specific-heat anomaly at elevated temperature. Comparison with the specific heat of $\mathrm{LaCu}_{2} \mathrm{Si}_{2}$ shows that neither phonons nor $5 d$ electrons contribute significantly to this anomaly. Instead, it indicates the removal of Zeeman degeneracies and perhaps also configurational degeneracies at the Ce sites and results from an interaction between the (unstable) $4 f$ shells and the conduction electrons. From the coefficient $\gamma$, a spin-fluctuation temperature, $T^{*} \simeq 10 \mathrm{~K}$, is estimated (cf. Bredl, Steglich, and Schotte ${ }^{4}$ ), which is in reasonable agreement with $T^{*}=20 \pm 10$ $\mathrm{K}$ from electronic transport $\mathrm{t}^{5}$ and inelastic neutron scattering ${ }^{10}$ experiments.

Around $0.5 \mathrm{~K}$ a second set of anomalies is observed: (i) below $T_{c}{ }^{(\rho)}=0.60 \mathrm{~K}$, the resistivity vanishes within the experimental resolution of $10^{-4}$, i.e., it becomes at least smaller than that of high-purity bulk Cu; (ii) at $T_{c}{ }^{(x)}=0.54 \mathrm{~K}$, the almost temperature-independent paramagnetic signal of the low-field ac susceptibility changes into a large diamagnetic signal; (iii) at $T_{c}{ }^{(C)}$ $=0.51 \mathrm{~K}$, the specific heat has a pronounced discontinuity $\Delta C=0.85 C_{n}\left(T_{c}\right)$ indicating a secondorder phase transition of $\mathrm{CeCu}_{2} \mathrm{Si}_{2}\left(C_{n}\right.$ is the specific heat at $T_{c}$ as extrapolated from higher temperatures). Clearly, the drop of $\rho$ and $\chi_{\text {ac }}$ indicates an onset of superconductivity. dc magnetization in low fields $\left(<10^{-4} \mathrm{~T}\right)$ was measured with aid of a SQUID magnetometer. Comparing the data obtained for the bulk $\mathrm{CeCu}_{2} \mathrm{Si}_{2}$ sample with those for a Cd sample of same geometry, we conclude that in this sample a net volume of the order of $10 \%$ exhibits the Meissner effect. We believe that this shows that the kind of superconductivity indicated by features (i) and (ii) cannot be related to the spurious phases (with total amount $<4$ vol \%). We note that the $\mathrm{LaCu}_{2} \mathrm{Si}_{2}$ sample does not show similar anomalies and, according to a $\chi_{\text {ac }}$ measurement, remains in the normal state at least down to $50 \mathrm{mK}$.

In order to check whether the specific-heat jump marks the onset of superconductivity or perhaps of antiferromagnetism [like in $\mathrm{CeAl}_{2}$ (Ref. 4)] we have repeated the measurements at $B=0$ and for two values of the external field, namely, 0.5 and $1 \mathrm{~T}$. Absolute and reduced values of the transition temperatures are collected in Table I. The reduced transition temperatures determined by different techniques are almost identical and vary linearly with magnetic field. This suggests that the specific-heat jump (i) has the same origin as the drop of both $\rho$ and $\chi_{\text {ac }}$ and (ii) cannot be attributed to an antiferromagnetic phase transition, because the latter requires a vertical slope of the critical field at the Neel temperature. Our experiments, therefore, lead to the conclusion that $\mathrm{CeCu}_{2} \mathrm{Si}_{2}$ becomes superconducting below $0.5 \mathrm{~K}$. The values of the critical field show type-II behavior.

We now discuss some observations which complicate the interpretation of our results: (i) Some spurious superconductivity $\left(T_{c}=0.2-0.3 \mathrm{~K}\right)$ was detected through a broad and tiny step in the $\chi_{\text {ac }}(T)$ curve for one $\mathrm{CeCu}_{2} \mathrm{Si}_{2}$ sample as well as one $\mathrm{LaCu}_{2} \mathrm{Si}_{2}$ sample. The origin of these effects is not yet clear. It can hardly be related to $C u \mathrm{Si}$ precipitations of either hcp or fcc structure (so far the only known superconducting materials formed with $\mathrm{Ce}, \mathrm{Cu}$, and $\mathrm{Si}$ ), because their transition temperatures are much too low, i.e., $T_{c}$ $=50-58 \mathrm{mK}$, Luo and Andre ${ }^{11}$ and $\$ 20 \mathrm{mK}$, Hoyt, ${ }^{12}$ respectively. (ii) There are aging effects, namely, an increase of $T_{c}{ }^{(\rho)}$ by $\simeq 30 \mathrm{mK}$, a corresponding decrease of $T_{c}{ }^{(C)}$ (see Table I) and a reduction of the ratio $\Delta C / C_{n}\left(T_{c}\right)$ from 0.85 to

TABLE I. Transition temperatures $T_{c}{ }^{(\rho)}, T_{c}{ }^{(\mathrm{X})}, T_{c}{ }^{(C)}$ at $B=0,0.5$, and $1 \mathrm{~T}$ as obtained in repeated experiments. Also given are the reduced transiton temperatures $t_{c}{ }^{(i)}=T_{c}{ }^{(i)} / T_{c}{ }^{(i)} \quad(B=0)$.

\begin{tabular}{ccccccc}
\hline \hline$B(\mathrm{~T})$ & $T_{c}^{(\rho)}(\mathrm{K})$ & $t_{c}^{(\rho)}$ & $T_{c}^{(\mathrm{X})}(\mathrm{K})$ & $t_{c}{ }^{(\mathrm{X})}$ & $T_{c}{ }^{(C)}(\mathrm{K})$ & $t_{c}^{\left({ }^{(C)}\right.}$ \\
\hline 0 & 0.63 & 1 & 0.54 & 1 & 0.49 & 1 \\
0.5 & 0.58 & 0.92 & 0.50 & 0.93 & 0.45 & 0.92 \\
1 & 0.53 & 0.85 & 0.45 & 0.83 & 0.41 & 0.84 \\
\hline
\end{tabular}


0.76 as found when the experiment was repeated after about two months during which the sample was kept under argon atmosphere. These observations indicate that presently the metallurgy of our samples is delicate and needs to be improved in the future.

Recently, we have prepared two $\mathrm{CeCu}_{2} \mathrm{Si}_{2}$ samples of the same quality as indicated before, which show a somewhat reduced coefficient $\gamma$ and a specific-heat jump of BCS size, i.e., $\Delta C / C_{n}\left(T_{c}\right)$ $\simeq 1.4$ (inset of Fig. 2). So far, one of these samples was studied by dc magnetization; a net volume of $\simeq 30 \%$ was found to exhibit the Meissner effect. With this same sample we have checked the influence of annealing on the specific-heat jump and found an increase of both the transition temperature (by $30 \mathrm{mK}$ ) and the transition width (by $50 \%)$ as well as a slight $(\simeq 10 \%)$ reduction of the jump height. Although our preliminary results with these new samples form a quite convincing case, we would welcome a confirmation by other workers of our conclusion that $\mathrm{CeCu}_{2} \mathrm{Si}_{2}$ is an intrinsic superconductor.

To summarize, $\mathrm{CeCu}_{2} \mathrm{Si}_{2}$ shows unstable- $4 f$ shell behavior. Well below $T^{*}=10 \mathrm{~K}$, a large $\gamma T$ term predominates the specific heat. We interpret this term as being due to very heavy fermion quasiparticles with degeneracy temperature $T_{\mathrm{F}}$ $\simeq T^{*}$. Below $0.5 \mathrm{~K}$, a large fraction of $\mathrm{CeCu}_{2} \mathrm{Si}_{1}$ becomes superconducting, in contrast to $\mathrm{LaCu}_{2} \mathrm{Si}_{2}$ which remains in the normal state at least down to $50 \mathrm{mK}$. The size of the specific-heat jump at $T_{c}$, in proportion to $\gamma T_{c}$, suggests that Cooperpair states are formed by these heavy fermions. Since the Debye temperature, $\theta$, is of the order of $200 \mathrm{~K},{ }^{5}$ we find $T_{c}<T_{\mathrm{F}}<\theta$ with $T_{c} / T_{\mathrm{F}} \simeq T_{\mathrm{F}} / \theta$ $\simeq 0.05$. This suggests that $\mathrm{CeCu}_{2} \mathrm{Si}$ (i) behaves as a "high-temperature superconductor" and (ii) cannot be described by conventional theory of superconductivity which assumes a typical phonon frequency $k_{\mathrm{B}} \Theta / h \ll k_{\mathrm{B}} T_{\mathrm{F}} / h$, the characteristic frequency of the fermions.

It is a great pleasure to thank the many people who have assisted with this work: V. Klink for preparing the samples, W. Assmus (Universität Frankfurt am Main) and G. Weimann (Femmeldetechnische Zentralamt, Darmstadt) for performing the microprobe analyses, S. Horn and the $\mathrm{ZCH} /$ Kernforschungsanlage Jülich for performing the x-ray analysis, A. C. Mota for advising us on the dc magnetization measurements, F. M. Mueller, D. Rainer, and K. D. Schotte for reading the manuscript and contributing many useful suggestions, P. F. de Châtel, B. Elschner,
E. Müller-Hartmann, E. Umlauf, and D。Wohlleben for stimulating and critical discussions.

This work was supported by the Deutsche Forschungsgemeinschaft under the auspices of Sonderforschungsbereich 65 Frankfurt am MainDarmstadt and Sonderforschungsbereich 125 Aachen-Jülich-Köln.

${ }^{1}$ B. T. Matthias, H. Suhl, and E. Corenzwit, Phys. Rev. Lett. 1, 449 (1958); L. P. Gor' kov and A. I. Rusinov, Zh. Eksp. Teor Fiz. 46, 1363 (1964) [Sov. Phys. JETP 19, 922 (1964)]; J. E. Crow, R. P. Guertin, and R. D. Parks, Phys. Rev. Lett. 19, 77 (1967); M. Peter, P. Donze, $\varnothing$. Fischer, A. Junod, J. Ortelli, A. Treyvaud, E. Walker, M. Wilhelm, and B. Hildenbrand, Helv. Phys. Acta 44, 345 (1971).

${ }^{2}$ M. Ishikawa and $\varnothing$. Fischer, Solid State Commun. $\underline{23}$, 37 (1977), and 24, 747 (1977); R. W. McCallum, D. C. Johnston, R. N. Shelton, W. A. Fertig, and M. B. Maple, Solid State Commun. 24, 501 (1977); W. A Fertig, D. C. Johnston, L. E. DeLong, R. W. McCallum, M. B. Maple, and B. T. Matthias, Phys. Rev. Lett. 38, 987 (1977); D. E. Moncton, D. B. McWhan, E. Corenzwit, J. Eckert, G. Shirane, and W. Thomalinson, Phys. Rev. Lett. $\underline{39}$, 1164 (1977); H. R. Ott, W. A. Fertig, D. C. Johnston, M. B. Maple, and B. T. Matthias, J. Low. Temp. Phys. 33, 159 (1978).

${ }^{3}$ E. Müller-Hartmann and J. Zittartz, Phys. Rev. Lett. 26, 428 (1971); G. Riblet and K. Winzer, Solid State Commun. 9, 1663 (1971), and Solid State Commun. 11, 175 (1971); M. B. Maple, W. A. Fertig, A. C. Mota, D. Wohlleben and R. Fitzgerald, Solid State Commun. 11, 829 (1972); F. Steglich and H. Armbrüster, Solid State Commun. 14, 903 (1974); S. D. Bader, N. E. Phillips, M. B. Maple, and C. A. Luengo, Solid State Commun. 16, 1263 (1975); K. Winzer, Solid State Commun. 24, $5 \overline{51}$ (1977).

${ }^{4}$ B. Barbara, J. X. Boucherle, J. L. Buevoz, M. F. Rossignol, and J. Schweizer, Solid State Commun. 24, 481 (1977); A. Benoit, J. Flouquet, and M. Ribault, J. Phys. (Paris), Lett. 39, L63 (1978); A. Benoit, J. Flouquet, M. Ribault, F. Flouquet, G. Chouteau, and R. Tournier, J. Phys. (Paris), Lett. 39, L94 (1978); C. D. Bredl, F. Steglich, and K. D. Schotte, Z. Phys. B 29,327 (1978); H. Armbrüster, F. Steglich, Solid State Commun. 27, 873 (1978); F. Steglich, C. D. Bredl, M. Loewenhaupt, and K. D. Schotte, J. Phys. (Paris), Colloq. 40, C5-301 (1979).

${ }^{5}$ W. Franz, A. Griessel, F. Steglich, and D. Wohlleben, Z. Phys. B 31,7 (1978).

${ }^{6}$ F. Steglich, in Proceedings of the Topical Meeting on Unusual Conditions of Superconductivity and Itinerant Magnetism in $d$-Materials, Bad Honnef, Germany, May, 1979 (unpublished).

${ }^{7}$ K. Andres, J. E. Graebner, and H. R. Ott, Phys. Rev. Lett. 27, 1779 (1975).

${ }^{8}$ This is also inferred from high-temperature measurements on $\mathrm{CeCu}_{2} \mathrm{Si}_{2}$ of both susceptibility [B. C. Sales and R. Viswanathan, J. Low Temp. Phys. 23, 449 
(1976)] and transport properties (Franz, Griessel, Steglich, and Wohlleben, Ref. 5).

${ }^{9}$ M. H. van Maaren, K. H. J. Buschow, and H. J. van Daal, Solid State Commun. 9 , 1981 (1971).

${ }^{10}$ E. Holland-Moritz, Ph.D. dissertation, University of Cologne, 1978 (unpublished).

${ }^{11}$ H. L. Luo and K. Andres, Phys. Rev. B 1, 3002 (1970).

${ }^{12}$ R. F. Hoyt, Ph.D. thesis, University of California, San Diego, 1978 (unpublished)。

\section{Excitonic Effects in Core-Hole Screening}

P. M. Th. M. van Attekum and G. K. Wertheim

Bell Laboratories, Murray Hill, New Jersey 07974

(Received 14 September 1979)

It is shown that the $\mathrm{C} 1 s$ core-electron line shape of graphite in photoemission is determined by an excitonic state near the Fermi energy in the hole-state density of states.

The observation (see Fig. 1) that the carbon $1 s$ photoemission line of graphite is strongly asymmetrical is difficult to understand from the point of view of many-body screening because the density of states at the Fermi energy $\left(E_{\mathrm{F}}\right)$ is very small compared with that of the simple metals. The additional fact that the shape of the line is well represented over a range of a few electron volts by the many-body power law only serves to deepen the puzzle since that shape is thought to be applicable only to the simple metals, i.e., those in which the density of states (DOS) remains constant near $E_{\mathrm{F}}$. In graphite the DOS at $E_{\mathrm{F}}$ is very small and initially rises linearly in both directions. The resolution of this dilemma requires an extension of the usual treatment of the many-body screening formalism.

In the sophisticated treatment of the many-body phenomenon the behavior is studied only in the

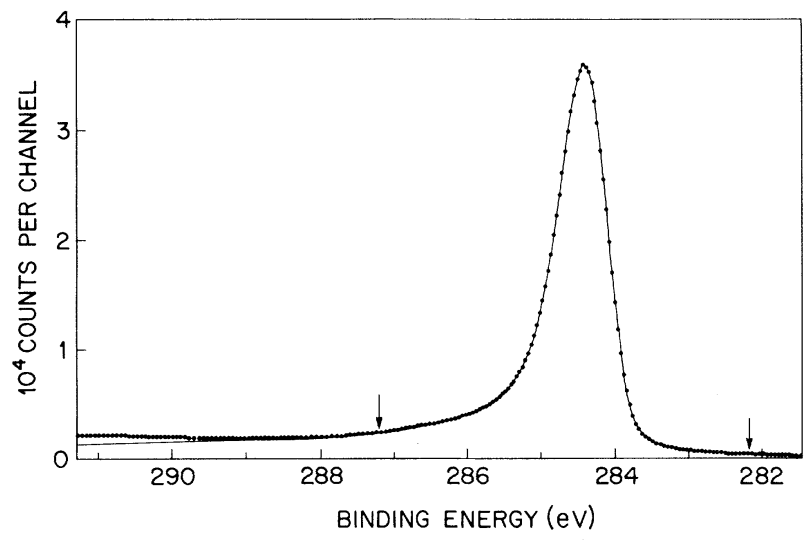

FIG. 1. X-ray photoemission spectrum of the C $1 s$ electrons of highly oriented pyrolytic graphite. The solid line represents a least-squares fit to the data between the arrows (see text for details). limit of small excitation energy., 2 The DOS, $g$, is assumed to be well behaved (i.e., no singularity) at $E_{\mathrm{F}}$ so that only $g\left(E_{\mathrm{F}}\right)$ enters explicitly into the many-body line shapes. Using the approach due to Hopfield ${ }^{3}$ it can be shown ${ }^{4}$ that the joint density of states (JDOS) for electron-hole pair excitation plays a crucial role in determining the line shape. For a well-behaved DOS the JDOS rises linearly for small excitation energy. Such a linear rise translates directly into the manybody line shape

$$
I(\omega) \propto\left[\left(\omega-\omega_{0}\right) / \xi\right]^{\alpha-1} .
$$

For simple metals it has been shown ${ }^{5}$ that this behavior is realized over a range of $\sim E_{\mathrm{F}} / 4$, as one would expect on the basis of a calculated JDOS. In metals with highly structured DOS more detailed calculations using a JDOS obtained from the actual band structure are required. ${ }^{4}$ For graphite this approach was tried with two forms of the band structure, those shown by Weinberger et $a l .{ }^{6}$ and by Dresselhaus, Dresselhaus, and Fischer. ${ }^{7}$ The latter has more detailed structure in the vicinity of $E_{\mathrm{F}}$. Neither one gives a line shape resembling that of graphite, largely because the JDOS is small and has strong positive curvature for small excitations.

The data shown in Fig. 1 were taken with a HP $5950 \mathrm{~A}$ spectrometer on a piece of vacuum cleaved graphite, highly oriented pyrolitic graphite (HOPG), grade ZYB, obtained from Union Carbide. Other forms of graphite give similar results. The data were fitted over a range from 282.2 to $287.2 \mathrm{eV}$ with the power-law line shape, by use of the equation of Doniach and Sunjic, ${ }^{8}$ and a closed-form expression for the instrumental resolution function. The resulting line is shown over full range of the data up to the $6-\mathrm{eV}$ plas- 